\title{
NUMB and NUMBL differences in gene regulation
}

\author{
José Manuel García-Heredia ${ }^{1,2,3}$ and Amancio Carnero ${ }^{1,3}$ \\ ${ }^{1}$ Instituto de Biomedicina de Sevilla, IBIS, Hospital Universitario Virgen del Rocío, Universidad de Sevilla, Consejo Superior \\ de Investigaciones Científicas, Seville, Spain \\ ${ }^{2}$ Department of Vegetal Biochemistry and Molecular Biology, University of Seville, Seville, Spain \\ ${ }^{3}$ CIBER de Cáncer, Instituto de Salud Carlos III, Pabellón 11, Planta 0, Madrid, Spain \\ Correspondence to: Amancio Carnero, email: acarnero-ibis@us.es
}

Keywords: NUMB; NUMBL; cancer; Notch pathway; WNT pathway

Received: September 06, $2017 \quad$ Accepted: January 03, $2018 \quad$ Published: January 11, 2018

Copyright: García-Heredia et al. This is an open-access article distributed under the terms of the Creative Commons Attribution License 3.0 (CC BY 3.0), which permits unrestricted use, distribution, and reproduction in any medium, provided the original author and source are credited.

\section{ABSTRACT}

NUMB, and its close homologue NUMBL, behave as tumor suppressor genes by regulating the Notch pathway. The downregulation of these genes in tumors is common, allowing aberrant Notch pathway activation and tumor progression. However, some known differences between NUMB and NUMBL have raised unanswered questions regarding the redundancy and/or combined regulation of the Notch pathway by these genes during the tumorigenic process. We have found that NUMB and NUMBL exhibit mutual exclusivity in human tumors, suggesting that the associated tumor suppressor role is regulated by only one of the two proteins in a specific cell, avoiding duplicate signaling and simplifying the regulatory network. We have also found differences in gene expression due to NUMB or NUMBL downregulation. These differences in gene regulation extend to pathways, such as WNT or Hedgehog. In addition to these differences, the downregulation of either gene triggers a cancer stem cell-like related phenotype. These results show the importance of both genes as an intersection with different effects over cancer stem cell signaling pathways.

\section{INTRODUCTION}

NUMB and NUMBL, or NUMB-Like, belongs to a closely conserved family of proteins with important roles in a large variety of cellular processes ranging from cell adhesion to ubiquitination [1-7]. NUMB, the first gene in the family identified in Drosophila [8], has been suggested to play a role in asymmetric division, allowing cell differentiation [7]. Most research conducted to date has been focused on the role of NUMB, assuming that NUMBL performs the same functions, although NUMBL does not display an asymmetric distribution in cells during mitosis [9]. In addition, its expression is different during development, with ubiquitous NUMB expression and more restricted expression of NUMBL in the central nervous system [6, 9-11]. Knock-out experiments in mice have shown that, although $N U M B L$ deletion showed no differences during embryogenesis, the deletion of $N U M B$ or combined $N U M B / N U M B L$ deletion were embryonic lethal $[6,12,13]$. Together, these differences show that, although $N U M B$ and $N U M B L$ have a conserved structure and domains [14], the functional differences between the proteins must also be considered.

$N U M B$ and $N U M B L$ have been characterized as tumor suppressor genes [15-17], leading to Notch signaling pathway inhibition $[4,17]$ or p53 stabilization $[18,19]$. NUMB inhibits the Notch pathway through its interaction with ITCH and NICD (Notch IntraCellular Domain), labeling NICD for ubiquitination and degradation [4, 20-22]. Although this is one of the most known roles of NUMB, this protein has also been linked to the WNT pathway, promoting $\beta$-catenin degradation through polyubiquitination [23]. The role of NUMB as a tumor suppressor gene has been widely characterized, revealing that lower NUMB levels are associated with a worse prognosis in malignant pleural mesothelioma [24]. In addition, different tumors, such as breast cancer, salivary gland carcinoma, non-small-cell lung carcinoma or medulloblastoma, also exhibit a downregulation of NUMB expression [25-28]. Conversely, NUMB 
overexpression reduces cell proliferation and increases cell sensitivity to cisplatin [24, 25, 29].

Previous results obtained by $N U M B L$ knockdown by shRNA, with no changes in $N U M B$ levels, showed an increment in tumorigenic properties and increased resistance to chemotherapy, with a worse prognosis in breast, lung and colorectal tumors [17]. Importantly, the downregulation of NUMBL also triggers Notch pathway activation, further increasing the epithelia-mesenchymal transition (EMT), cancer stem cell (CSC) transcriptional markers and CSClike phenotypes. $N U M B L$ has also been described as a tumor suppressor gene, mainly based on its ability to inhibit the Notch pathway $[17,30,31]$. However, NUMBL can also activate Hedgehog signaling, which represents a functional difference compared with NUMB [32]. According to these results, NUMBL can activate Hedgehog signaling and thus increase the stem cell population. This phenomenon suggests that, under certain circumstances, NUMBL could act as an oncogene. This process has also recently been described for NUMB, showing that an altered isoform expression is common in cancer cells [23, 33-35].

A small percentage of human tumors exhibit lower NUMBL expression than normal tissue, being this reduced expression associated with a poor prognosis and worse patient survival $[30,31]$. Inhibition of only one NUMB family protein is sufficient to modify cancer cell properties, since a partial decrease in NUMB or NUMBL is sufficient to increase Notch pathway activation and cancer stem-like properties. This phenomenon suggests that NUMB and NUMBL act as essential regulators of cancer cell properties, individually acting in a dosedependent manner and regulating the same pathway with a certain degree of redundancy. Like NUMB, NUMBL seems to regulate Notch pathway activity $[36,37]$. It is interesting to note that the downregulation of only one of these proteins, either NUMB or NUMBL, is sufficient to allow Notch pathway activation, increasing the pool of CSC-like cells [38-41]. However, whether there is a dose effect of combined NUMB/NUMBL or whether the downregulation of both proteins may coexist in some very aggressive tumors remains unknown. Such an additive effect still must be demonstrated, as well as whether the accumulative effects are linear or reach a certain threshold with no further increases in tumorigenicity. Finally, the effects of the distinct tumor suppressor activities on different pathways in cells must be elucidated.

Here, we showed the mutual exclusivity of NUMB and NUMBL in tumors and the opposite gene regulation affecting at least three different signaling pathways: Notch, WNT and Hedgehog. Although both genes equally regulate Notch, the WNT and Hedgehog pathways are oppositely regulated by NUMB and NUMBL, according to our gene transcription analysis. However, the final phenotypic endpoint induced by $N U M B$ or $N U M B L$ downregulation was similar, suggesting some hierarchy in the signaling pathways.

\section{RESULTS}

\section{$N U M B$ and $N U M B L$ show a negative correlation in tumors}

As mentioned above, NUMB and NUMBL are commonly described as proteins with a very similar function, characterized as tumor suppressors. To identify any differential effects in tumors, we analyzed a total of 95 datasets in ten different tumors (Acute Lymphoblastic Leukemia, Acute Myeloid Leukemia, Chronic Lymphocytic Leukemia, ovarian colon/rectal, lung, renal breast, neuroblastoma or esophageal tumors) using R2 software. We observed a clearly marked trend between $N U M B$ and $N U M B L$ since were negatively correlated in 72 of the 95 considered datasets (Figure 1A). Indeed, the correlation of $N U M B$ and $N U M B L$ expression provided a significant Pearson value $\left(R=-0.355, p=4.4 \times 10^{-4}\right)$ (Figure $\left.1 \mathrm{~B}\right)$, as clearly shown in the heat maps of tumors from colon, lung, breast, endometrium or kidney, in which individual samples can be compared (Figure 1C). These results, which were obtained directly from human tumor samples, showed that when one of these genes was highly expressed in tumors, the other was expressed at a lower rate. In fact, we also explored datasets derived from normal samples. We found that the negative correlation of $N U M B / N U M B L$ was also present in non-tumoral samples, showing that both genes are to some extent mutually exclusive in cells (Supplementary Figure 1). This result suggests a stringent gene regulation to avoid undesirable effects. These differences might be due to differences in gene methylation, allowing higher expression of one of the genes in cells. To explore this phenomenon, we looked for changes in NUMB or NUMBL methylation using TCGA Wanderer resource [42]. By doing this, we found that $N U M B$ is highly methylated in tumors regarding control (Figure 2A). However, the opposite happens with $N U M B L$, being highly methylated in normal samples but not in tumoral samples (Figure 2B). Differences in $N U M B$ or $N U M B L$ methylation between normal and tumoral samples appear to be general, as can be deduced from the fact that a common patron is obtained using four different CG probes (Figure 2C). At that way, for $N U M B$, methylation is higher in tumoral samples regarding control samples, while for $N U M B L$ occurs the opposite. In addition, if we focus in the probe $\operatorname{cg} 01582648$, the percentage of tumoral samples with a methylation beta value higher than 0.8 is significantly higher regarding normal samples in breast invasive carcinoma ( $80.9 \%$ vs. $33.3 \%$ ), colon adenocarcinoma ( $69.8 \%$ vs. $5.2 \%$ ) and lung adenocarcinoma ( $88.8 \%$ vs. $71.6 \%$ ) (Figure 2D). Again, the opposite happen for $N U M B L$ probe $\operatorname{cg} 20525355$, being tumoral samples lower methylated regarding normal samples in colon adenocarcinoma (61\% vs. $94.7 \%)$ and lung adenocarcinoma ( $75.8 \%$ vs. $100 \%$ ) (Figure 2E). These results point to the possibility that $N U M B$ or $N U M B L$ 
might be preferentially expressed in a specific cell or tissue. Furthermore, suggest certain redundancy in the roles.

\section{$N U M B$ and $N U M B L$ have few common genes with the same correlations}

To identify the genes that correlated to $N U M B$ and/or $N U M B L$ in the same tumors, we performed the following analysis. We first selected public datasets from transcriptomic analyses of lung, cervical, breast and colorectal tumors (Supplementary Table 1). Next, we determined the genes that correlated to $N U M B$ or $N U M B L$ in all considered tumor types and then compared the individual tumor types to obtain a map of common genes correlating to $N U M B$ or $N U M B L$ expression in various tumors. We found 675 genes that were positively correlated and 691 genes that were negatively correlated to $N U M B L$ in at least three of the considered tumor types. Regarding $N U M B$, we found 350 genes that were positively correlated and 108 genes that were negatively correlated, taking into account at least three of the tumors (Figure 3, Supplementary Table 2). Due to the previously assumed overlapping functions of NUMB and NUMBL in cells, it would be expected to find genes that were positively or negatively correlated to $N U M B$ and $N U M B L$. In our bioinformatics analysis of tumors considering all tumor types, no common genes from public transcriptome tumor datasets were positively correlated both to $N U M B$ and $N U M B L$. However, we obtained a short list of 5 genes that were negatively correlated both to $N U M B$ and $N U M B L$ (as expected from its behavior as tumor suppressors), a second list of 21 genes that were positively correlated to $N U M B$ and negatively correlated to $N U M B L$, and two genes negatively correlated to $N U M B$ and positively correlated
A

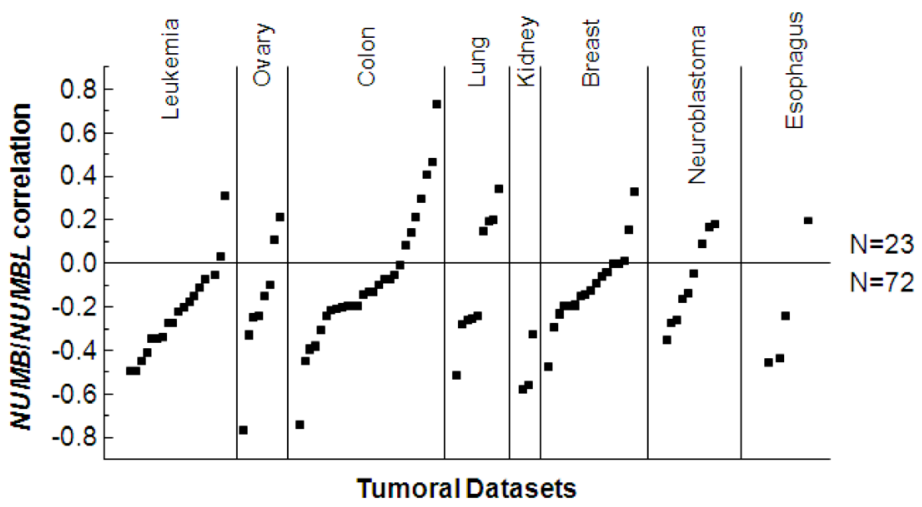

B

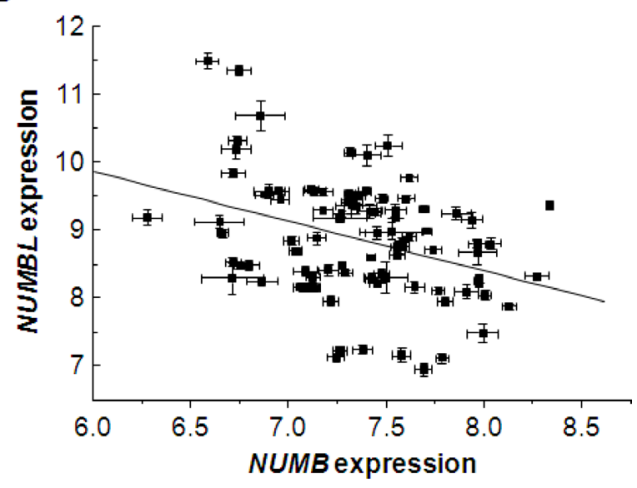

C

Tumor Colon Adenocarcinoma - TCGA-286

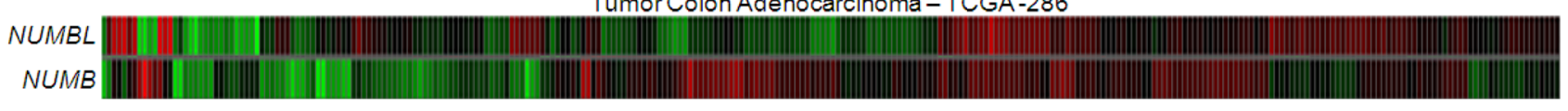

TumorLung-Bild - 114

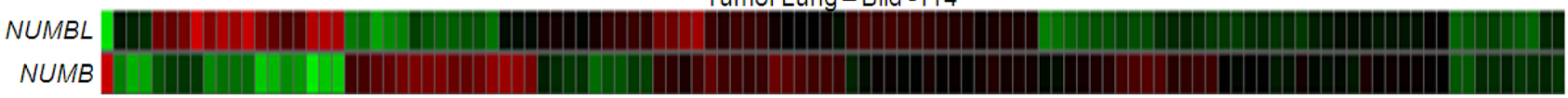

Tumor Breast- EXPO -351

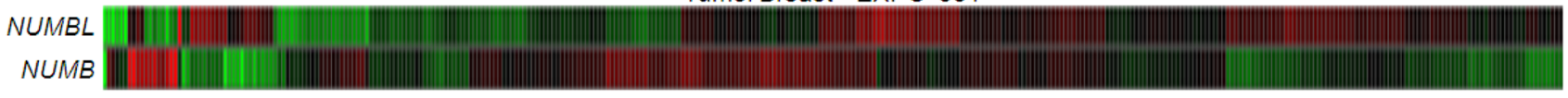

Tumor Endometrium - EXPO -209

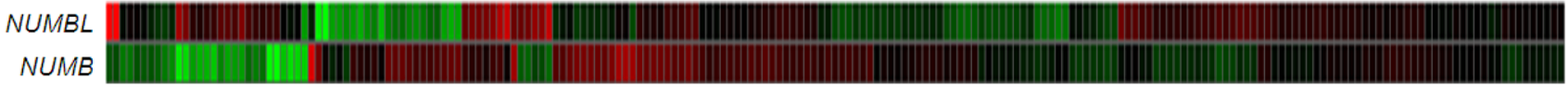

Tumor Kidney Renal Clear Cell Carcinoma - TCGA -533

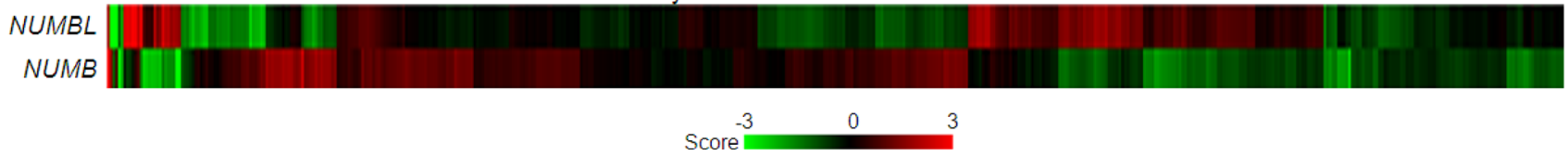

Figure 1: $N U M B$ and $N U M B L$ are negatively correlated in human tumors. (A) $N U M B / N U M B L$ correlations in leukemia, ovarian, colon, lung, kidney, breast, neuroblastoma and esophagus tumors, showing a higher percentage of negative correlations. (B) Correlation of $N U M B$ and $N U M B L$ expression in human tumors, showing a significant Pearson correlation coefficient $(R=-0.355$, $\left.p=4.4 \times 10^{-4}\right)$. (C) Heat map of $N U M B$ and $N U M B L$ expression in human tumors. 
to $N U M B L$ (Figure 4). However, when we compared the genes that were correlated to $N U M B$ or $N U M B L$ in each individual tumor type, we found common positively or negatively correlated genes, suggesting that NUMB and NUMBL participate in different gene regulatory processes, depending on the tissue (Supplementary Table 3).

\section{Gene ontology analysis of NUMB and/or NUMBL correlations}

Next, we performed Gene Ontology (GO)-term and Reactome enrichment analysis, considering separately the genes correlated to $N U M B$ or $N U M B L$, due to the low
A

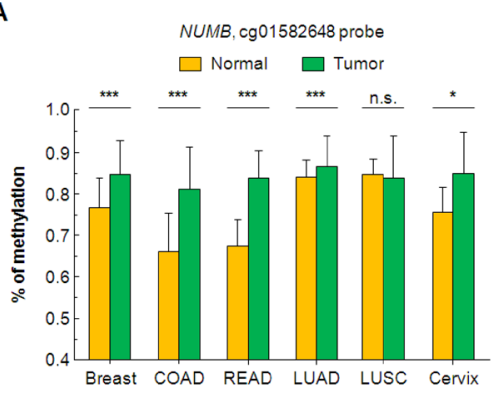

D

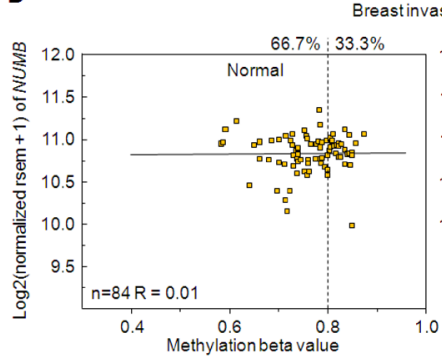

Colon adenocarcinom
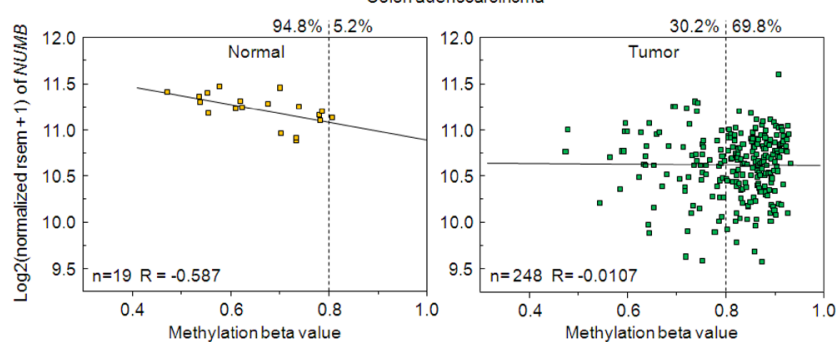

Lung adenocarcinoma
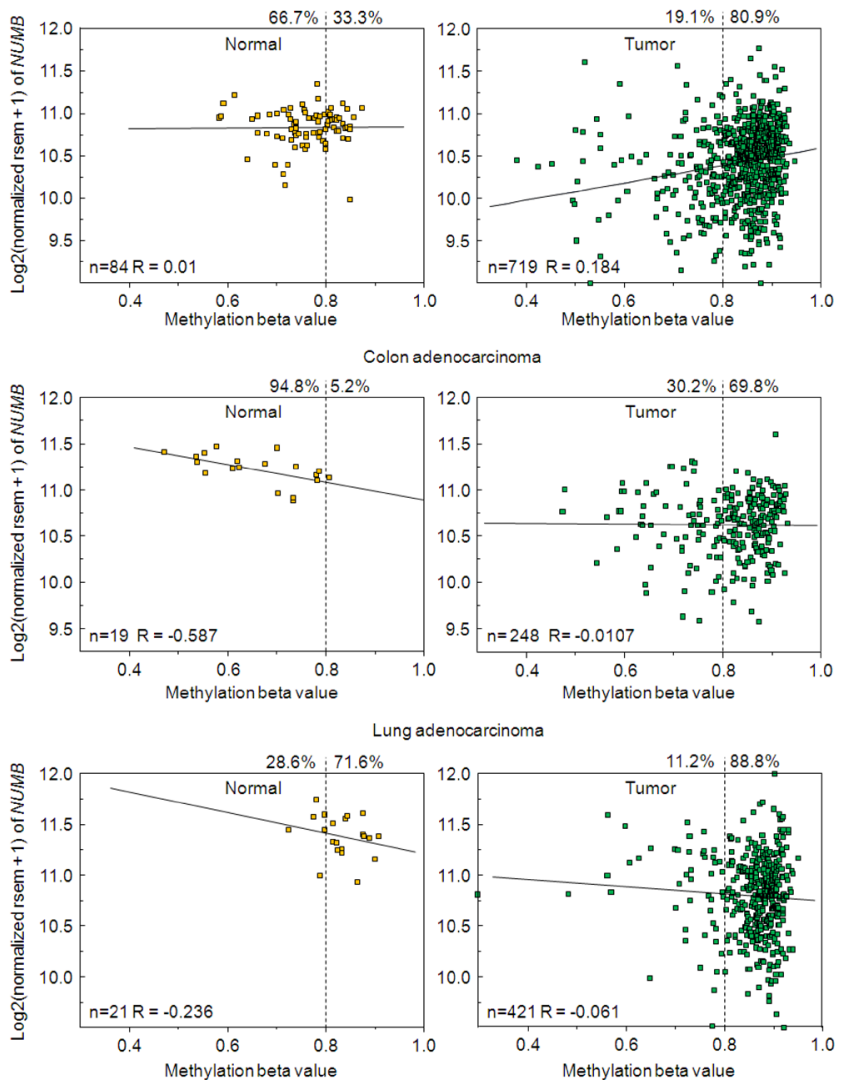

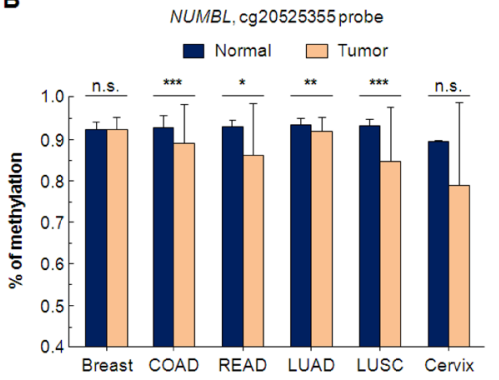

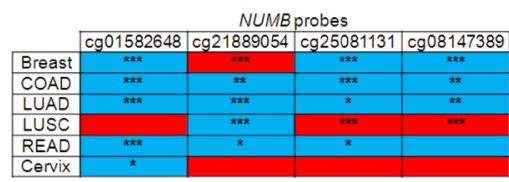

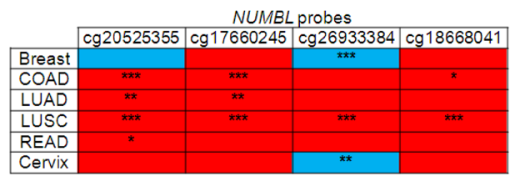

- Tumor/Normal<1 $\square$ Tumor/Normal>1

E
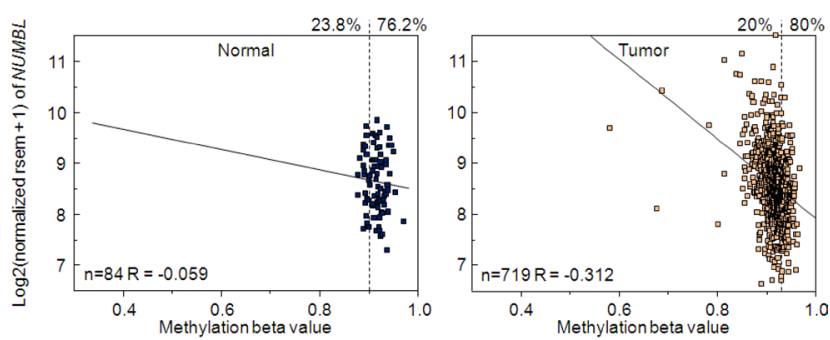

Colon adenocarcinome

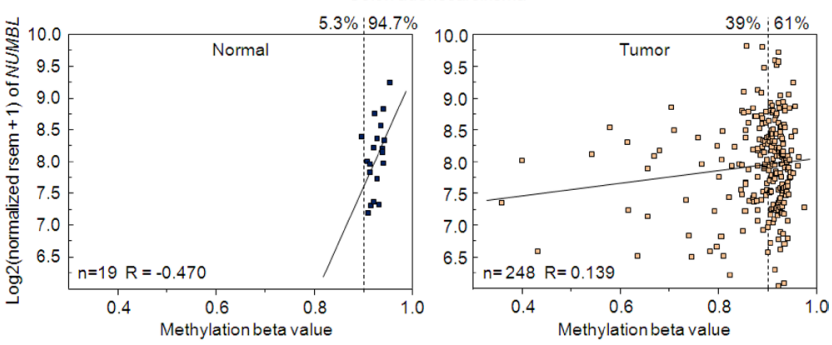

Lung adenocarcinoma
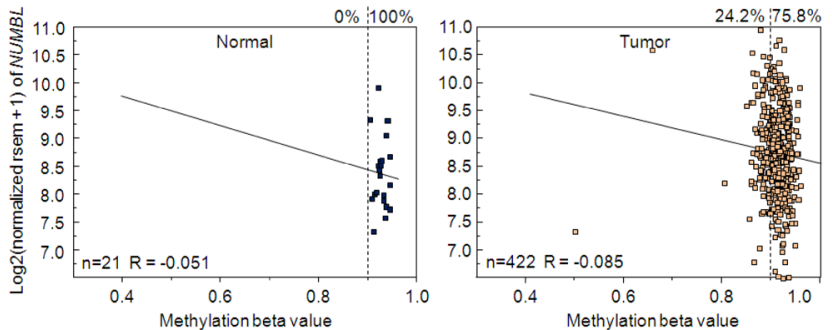

Figure 2: Changes in NUMB/NUMBL methylation in tumoral samples. (A) Percentage of $N U M B$ methylation for cg0158648 probe in six different tumors. Breast: Breast invasive carcinoma; COAD: Colon adenocarcinoma; READ: Rectum adenocarcinoma; LUAD: Lung adenocarcinoma; LUSC: Lung squamous cell carcinoma; Cervix: cervical squamous cell carcinoma and endocervical adenocarcinoma. (B) Percentage of NUMBL methylation for cg20525355 probe in six different tumors. (C) $N U M B$ and $N U M B L$ are differentially methylated in normal or tumoral samples. Red color: tumoral samples with a lower methylation value regarding normal samples. Blue color: tumoral samples with a higher methylation value regarding normal samples. (D) Relation between expression and methylation value for $N U M B$, using cg0158648 probe, in breast invasive carcinoma, colon adenocarcinoma and lung adenocarcinoma. (E) Relation between expression and methylation value for $N U M B L$, using cg20525355 probe, in breast invasive carcinoma, colon adenocarcinoma and lung adenocarcinoma. The Student's $T$ test was used to determine significant differences $\left({ }^{*}=p<0.05 ;{ }^{* *}=p<0.01\right.$; $\left.{ }^{* * *}=p<0.001\right)$. 
number of common genes (Supplementary Table 4-7). Interestingly, we obtained common GO and Reactome terms for genes negatively correlated with $N U M B$ or NUMBL (Supplementary Table 8). In particular, we found negative correlations with protein synthesis (from ribosome to mRNA maturation), general metabolism or protein transport. In addition, we found Reactome terms related to cell cycle, suggesting a possible deregulation due to changes in $N U M B$ or $N U M B L$ expression (Supplementary Table 8). At that way, one of the common genes negatively correlated both to $N U M B$ and $N U M B L$ was $C D K 4$, a known gene connected to cell division. In addition, we also found components (MCM3 for $N U M B L$ and $M C M 2$ for $N U M B$ ) of the minichromosome maintenance protein (MCM) complex, necessary for cell cycle transition to S-phase [43]. These results can be related to the functions of $N U M B$ and $N U M B L$ as negative

Positive correlations
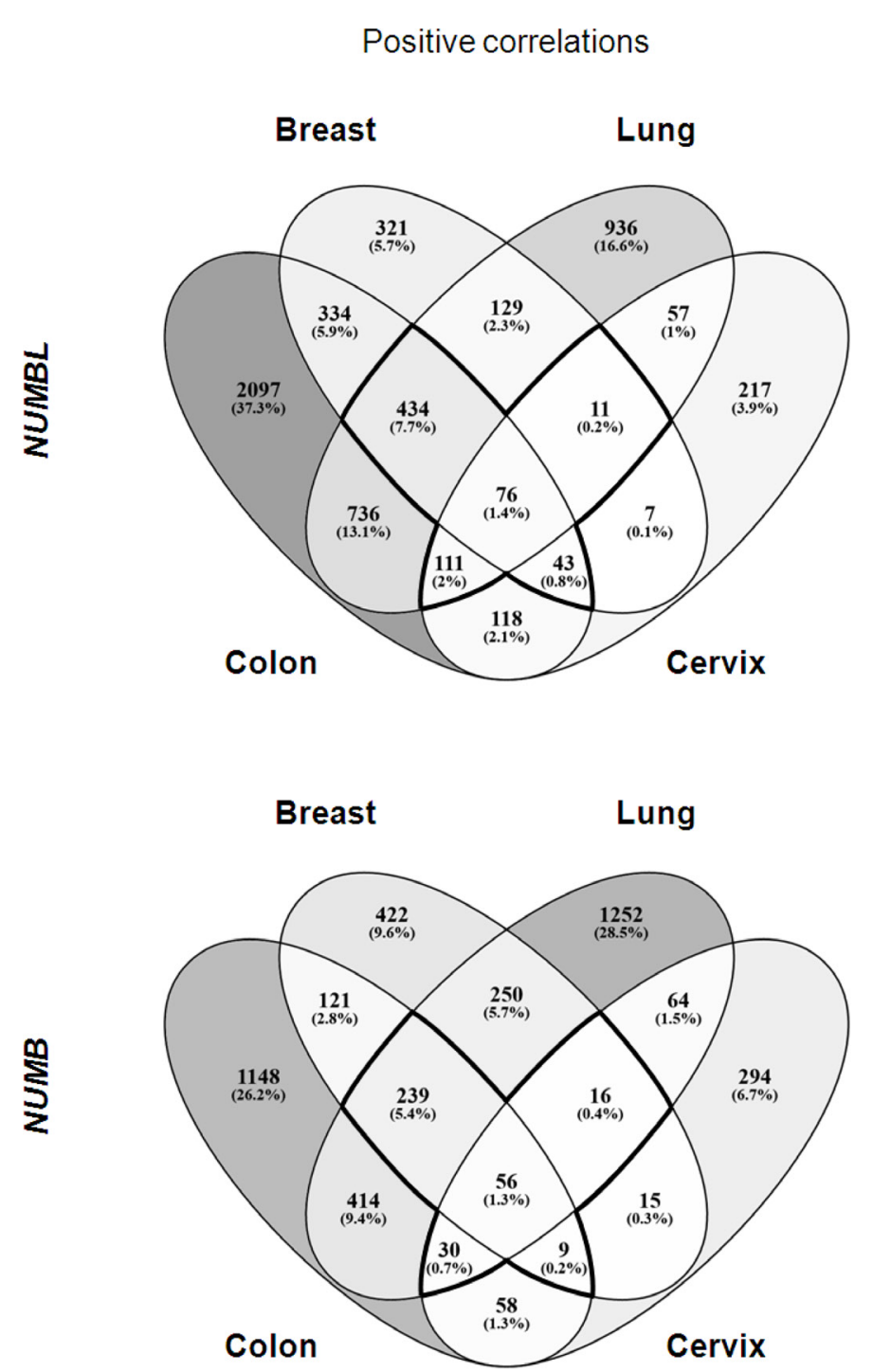

regulators of cell division, supporting their roles as tumor suppressor genes.

From the list of genes that positively correlated with either $N U M B$ or $N U M B L$, similar GO-terms could not be obtained. However, it is interesting to note that, regarding $N U M B$, some GO-terms were related to vesicle transport, a previously known $N U M B$ function [44, 45] (Table 1). However, $N U M B L$ appeared to be positively correlated to genes related to gene regulation and RNA metabolism (Table 2), suggesting an unexplored role for this gene.

Genes that were negatively correlated to $N U M B L$ resulted in a very extensive list of GO and Reactome terms, some of which were related to three different signaling pathways: NF-кB, WNT and Hedgehog, suggesting that, as previously reported [32], NUMBL could modulate the Hedgehog pathway (Table 3). We also found that $N U M B L$ was negatively correlated to NF-kB signaling,

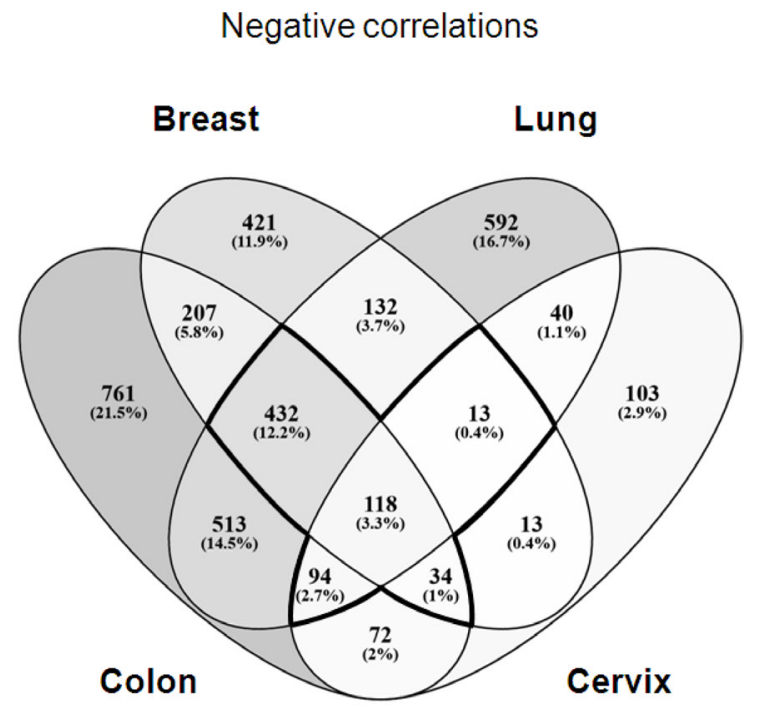

Figure 3: Venn diagram of genes positively and negatively correlated to $N U M B$ or $N U M B L$ in breast, lung, colon and cervix tumors $(p$-value $<\mathbf{0 . 0 5})$. 
mitochondrial tricarboxylic acid cycle (TCA) and p53 stabilization, as also previously described $[30,46,47]$.

\section{$N U M B$ and $N U M B L$ show opposite correlations in some signaling pathways}

NUMB and NUMBL have been previously described as inhibitors of the Notch pathway due to their interactions with NICD, allowing it to be labeled for ubiquitination and degradation. However, we did not find any GO or Reactome term clearly related to the Notch pathway, so we decided to search specifically for genes related to this pathway. We also searched for genes in the WNT and Hedgehog pathways since both pathways were negatively correlated with $N U M B L$ (Table 3). A significant percentage of genes related to the Notch pathway showed the same behavior according to the presence of $N U M B$ or NUMBL (Figure 5). Genes such as CFLAR, EP300, GBP2, HEYL, KLF7, NOTCH1 or POFUT showed a similar correlation with $N U M B$ or $N U M B L$ in the three tumor types considered (Figure 5). However, many of the genes correlating with $N U M B L$ showed an opposite behavior with $N U M B$ in the other two analyzed pathways, WNT and Hedgehog (Figure 5), independently of the type of tumor. These results indicated that $N U M B$ and $N U M B L$ could differentially modulate different signaling pathways in a very different fashion. These results also indicate that besides the previously observed exclusivity, the full redundancy does not exist between these two genes. Only in the Notch pathway, equal regulation is maintained.

To experimentally assess this opposite gene regulatory role, we decided to analyze gene expression using T47D and HeLa cells transfected with specific shRNAs against $N U M B$ or $N U M B L$ (Figure 6A). Focusing on genes in the Notch pathway, we observed an equivalent activation of this pathway due to the downregulation of $N U M B$ or $N U M B L$ (Figure $6 \mathrm{~B}$ ), correlating with previous reports $[17,48]$. Due to the relevance of the different gene associations with $N U M B$ and $N U M B L$, we extended our analysis to the other two signaling pathways, WNT and Hedgehog. As expected from the bioinformatics analysis, we found opposite results due to the downregulation of $N U M B$ or $N U M B L$ in the mRNA levels of the target genes $T C F 4, E N 1, S O X 9$ and BTRC, which are considered readouts of the WNT pathway (Figure 6C); or PTCH1, GLI1, GLI2 and $C D H 1$, considered read-outs of the Hedgehog pathway (Figure 6D).

Our bioinformatics results also led us to analyze other genes with opposite correlations in tumors, such as $N F \kappa B 1$, $E R B B 2$ and TEAD1. Measurement of the transcriptional responses of these genes to the reduced expression of $N U M B$ or $N U M B L$ revealed an opposite regulation of these genes in response to NUMB or NUMBL (Figure 6E), as expected based on our bioinformatics analysis. It is

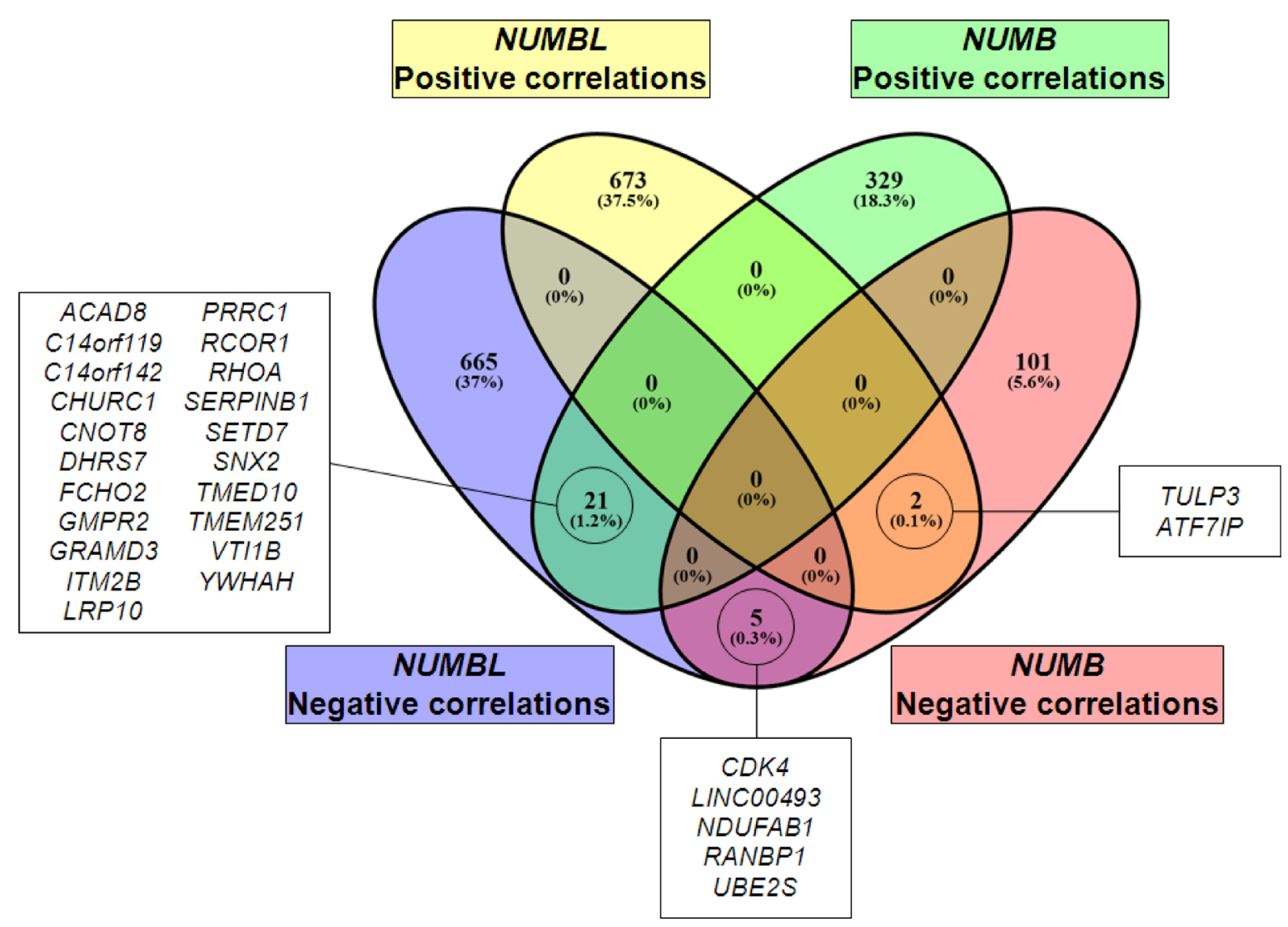

Figure 4: Venn diagram of genes positively or negatively correlated to $N U M B$ or $N U M B L$, showing coincident genes. 
Table 1: GO-terms obtained from genes positively correlated with $N U M B$

\begin{tabular}{lcc}
\hline GO-term & Fold enrichment & $\boldsymbol{p}$-value \\
\hline vesicle targeting & 8.47 & $2.76 \times 10^{-4}$ \\
COPII vesicle coating & 8.37 & 0.0161 \\
vesicle targeting, rough ER to cis-Golgi & 8.37 & 0.0161 \\
vesicle coating & 8.12 & 0.0207 \\
COPII-coated vesicle budding & 7.87 & 0.0264 \\
vesicle targeting, to, from or within Golgi & 7.76 & 0.0298 \\
actomyosin & 6.9 & 0.0372 \\
establishment of vesicle localization & 4.76 & $3.19 \times 10^{-4}$ \\
vesicle localization & 4.53 & $6.87 \times 10^{-4}$ \\
Golgi vesicle transport & 4.04 & $2.34 \times 10^{-4}$ \\
Vesicle organization & 3.47 & 0.0341 \\
vesicle-mediated transport & 2.27 & $5.75 \times 10^{-3}$ \\
Golgi subcompartment & 2.27 & 0.0332 \\
Golgi apparatus & 2.08 & $5.66 \times 10^{-4}$ \\
cytoplasmic vesicle & 1.85 & $4.58 \times 10^{-4}$ \\
intracellular vesicle & 1.85 & $4.82 \times 10^{-4}$ \\
vesicle & 1.53 & $1.58 \times 10^{-3}$ \\
\hline
\end{tabular}

Table 2: GO-terms obtained from genes positively correlated with $N U M B L$

\begin{tabular}{lcc}
\hline GO-term & Fold Enrichment & $\boldsymbol{p}$-value \\
\hline transcription, DNA-templated & 1.89 & $1.31 \times 10^{-8}$ \\
nucleic acid-templated transcription & 1.89 & $1.35 \times 10^{-8}$ \\
RNA biosynthetic process & 1.88 & $1.71 \times 10^{-8}$ \\
nucleobase-containing compound biosynthetic process & 1.72 & $1.94 \times 10^{-6}$ \\
DNA binding & 1.71 & $4.75 \times 10^{-5}$ \\
RNA metabolic process & 1.64 & $3.86 \times 10^{-6}$ \\
regulation of RNA metabolic process & 1.53 & $1.30 \times 10^{-4}$ \\
regulation of RNA biosynthetic process & 1.51 & $1.25 \times 10^{-3}$ \\
regulation of transcription, DNA-templated & 1.51 & $1.25 \times 10^{-3}$ \\
\hline
\end{tabular}

interesting to note that these three genes are part of other signaling pathways (NFKB, ERBB/HER and Hippo) [4951], likely suggesting complex interaction of NUMB/L proteins with the signaling network.

\section{$N U M B$ and $N U M B L$ downregulation equally increases stem cell properties}

Although $N U M B$ and $N U M B L$ downregulation act differently regarding some of the considered genes, suggesting that $N U M B$ and $N U M B L$ target some different pathways, the global effect caused by their downregulation is very similar. To explore this point, we decided to measure the expression of some genes associated with the acquisition of stem cell-like properties and observed a clear increment in $S O X 2, B M I 1$ and $N A N O G$ genes in response to both $N U M B$ and $N U M B L$ downregulation (Figure 7A), which have been previously related to stem cell properties [52-54]. The increment in the transcription of stem celllike genes, together with the previously described increment in other stem cell-like genes related to $N U M B$ or $N U M B L$ downregulation, such as OCT4 or KLF4, showed that, for all analyzed conditions, the cells acquire a more dedifferentiated state, typical of stem-like cells [17, 48].

To rate these stem cell-like properties, we cultured cells at a low density, so that individual clones formed independent colonies. These clones can be classified as holoclones, meroclones and paraclones, according to their ability to reconstitute a tumor $[55,56]$. Holoclones are considered to derive from cells with stem cell-like properties. We found that either $N U M B$ or $N U M B L$ downregulation caused similar increments in the 
Table 3: GO and Reactome pathways terms related to Hedgehog, WNT and NF-kB pathways, obtained from genes negatively correlated with $N U M B L$

\begin{tabular}{lcc}
\hline GO-term & Fold enrichment & p-value \\
\hline NIK/NF-kappaB signaling & 5.15 & $3.62 \times 10^{-3}$ \\
respiratory electron transport chain & 4.55 & $4.37 \times 10^{-5}$ \\
Wnt signaling pathway, planar cell polarity pathway & 4.68 & $5.27 \times 10^{-3}$ \\
\hline Reactome pathways term & Entities p-Value & Entities FDR \\
\hline Hedgehog 'off' state & $1.72 \times 10^{-3}$ & 0.0137 \\
GLI3 is processed to GLI3R by the proteasome & $3.06 \times 10^{-6}$ & $9.18 \times 10^{-5}$ \\
Degradation of GLI2 by the proteasome & $3.06 \times 10^{-6}$ & $9.18 \times 10^{-5}$ \\
Degradation of GLI1 by the proteasome & $3.06 \times 10^{-6}$ & $9.18 \times 10^{-5}$ \\
Hedgehog ligand biogenesis & $1.74 \times 10^{-5}$ & $2.44 \times 10^{-4}$ \\
Hh mutants that don't undergo autocatalytic processing are degraded & $1.16 \times 10^{-5}$ & $2.06 \times 10^{-4}$ \\
by ERAD & $2 \times 10^{-5}$ & $2.65 \times 10^{-4}$ \\
Hh mutants abrogate ligand secretion & $2.63 \times 10^{-3}$ & 0.021 \\
Beta-catenin independent WNT signaling & $1.7 \times 10^{-5}$ & $2.44 \times 10^{-4}$ \\
Degradation of beta-catenin by the destruction complex & $5.47 \times 10^{-6}$ & $1.26 \times 10^{-4}$ \\
Degradation of AXIN & $3.7 \times 10^{-6}$ & $9.62 \times 10^{-5}$ \\
Autodegradation of Cdh1 by Cdh1:APC/C & $2.72 \times 10^{-5}$ & $3.26 \times 10^{-4}$ \\
Dectin-1 mediated noncanonical NF-kB signaling & $6.81 \times 10^{-5}$ & $6.81 \times 10^{-4}$ \\
Activation of NF-kappaB in B cells & $2.4 \times 10^{-3}$ & 0.0192 \\
TNFR2 non-canonical NF-kB pathway & $1.16 \times 10^{-5}$ & $2.06 \times 10^{-4}$ \\
NIK->noncanonical NF-kB signaling & $2.58 \times 10^{-4}$ & $2.58 \times 10^{-3}$ \\
Citric acid cycle (TCA cycle) & $5.75 \times 10^{-9}$ & $9.03 \times 10^{-7}$ \\
The citric acid (TCA) cycle and respiratory electron transport & $1.69 \times 10^{-6}$ & $7.61 \times 10^{-5}$ \\
Stabilization of p53 & $1.26 \times 10^{-5}$ & $2.06 \times 10^{-4}$ \\
p53-Dependent G1 DNA Damage Response & & \\
\hline
\end{tabular}

percentages of holoclones of HeLa and T47D cells (Figure 7B). In addition, tumorspheres, colonies derived from stem cell-like cells that grow in suspension, showed a significant increment in number in both cell lines, demonstrating again that $N U M B / N U M B L$ downregulation increased stem cell-like properties (Figure 7C), regardless of the differences in pathway activation.

\section{DISCUSSION}

$N U M B$ and $N U M B L$ have been considered tumor suppressor genes with very similar functions because both can regulate the Notch pathway [5, 17, 22, 30, 57-59]. Our initial analysis in human tumors showed that both genes are negatively correlated in more than $75 \%$ percent of the considered datasets ( 72 out 95 different datasets). In addition, their expression in non-tumoral samples, which is close to $75 \%$, shows that this opposite regulation is a common effect in human cells. Their role as negative regulators of the Notch pathway likely requires a higher transcription of one of the genes than the other, allowing only one of the two proteins to effectively perform its regulatory role. This notion is supported by development studies conducted in mouse and chicken embryos, where NUMB is broadly expressed, while NUMBL appears to be enriched in the developing central nervous system [9-11].

Our previous results have focused on the downregulation of $N U M B$ or $N U M B L$ by shRNA [17, 48]. We showed that only that the downregulation of only $N U M B$ or $N U M B L$ causes an increment in the tumorigenic properties of cells, allowing us to focus on their roles as tumor suppressor genes. Therefore, cells with lower NUMBL levels are more resistant to drugs that are commonly used in cancer chemotherapy, such as doxorubicin or vincristine [17]. In fact, loss of NUMBL protein has been associated with an increase in metastasis and worse survival [60].

Our bioinformatics analysis allowed us to obtain an unexpected network of gene correlations with $N U M B$ or $N U M B L$. Due to their accepted roles as genes with similar functions, we expected to find common correlations for both genes in different tumors. However, our analysis showed that $N U M B$ and $N U M B L$ exhibited 
almost independent correlations when we considered the four tumor types. This result could be due to different regulatory mechanisms, depending on the tissue in which $N U M B$ or $N U M B L$ is expressed. A comparison of each tumor type allowed us to identify a small number of genes that were equally related to $N U M B$ and $N U M B L$, different genes related to similar functions, and genes with differential expression. GO-terms obtained with the different lists of genes that were correlated to $N U M B$ described functions such as endocytic protein with important roles in protein transport [2, 3, 44, 45]. GOterms related to $N U M B L$ were related to gene transcription and some of its previously described roles [17, 31, 61]. In addition, common GO and Reactome terms obtained from negative correlations to $N U M B$ or $N U M B L$ proceeded from different list of genes, pointing to a different gene regulation network. These data may confirm the different cellular roles of each protein.

The Notch pathway is involved in the maintenance of tumor stemness and cancer metastasis. This pathway is activated in different tumors, such as lung, colon, breast and prostate tumors, and in sarcomas, melanomas, leukemias and lymphomas [58, 59, 62-65]. In addition, Notch activity has been linked to cancer metastasis by inducing EMT, tumor angiogenesis processes and anoikis resistance of tumor cells $[66,67]$. After its interaction with a ligand, Notch is proteolytically cleaved, and
NICD is released into the cytoplasm, allowing its nuclear translocation [68, 69]. We have recently showed that NUMBL behaves, such as NUMB, its close relative, as a tumor suppressor gene regulating the Notch pathway. Here, we showed that NUMB and NUMBL presented mutual exclusivity in tumors, suggesting that the tumor suppressor effect on the Notch pathway is regulated by one protein at a time, thus simplifying the regulatory network. In addition, $N U M B$ and $N U M B L$ also showed opposite correlations in non-tumoral samples, suggesting that this opposite relationship is a common effect in cells. However, the two proteins, which share approximately $55 \%$ of their amino acid sequence, did not show total redundancy, as deduced from the differences in mRNA

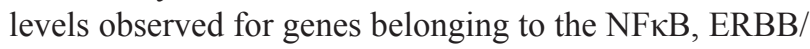
HER, Hippo, WNT and Hedgehog pathways. Thus, our results obtained by downregulation of $N U M B L$ are consistent with previously published studies showing that NUMBL negatively influences the NFאB pathway and positively affects the Hedgehog pathway [31, 32]. In addition, although GLI1 and GLI2 should have similar behaviors, considering their transcription is activated by Hedgehog signaling and appear to be downregulated when the Hedgehog pathway is inactive, GLI1 is also connected to Notch signaling through HES1 inhibition [70, 71]. The large number of genes that are transcriptionally affected in an opposite manner suggests that NUMB and NUMBL
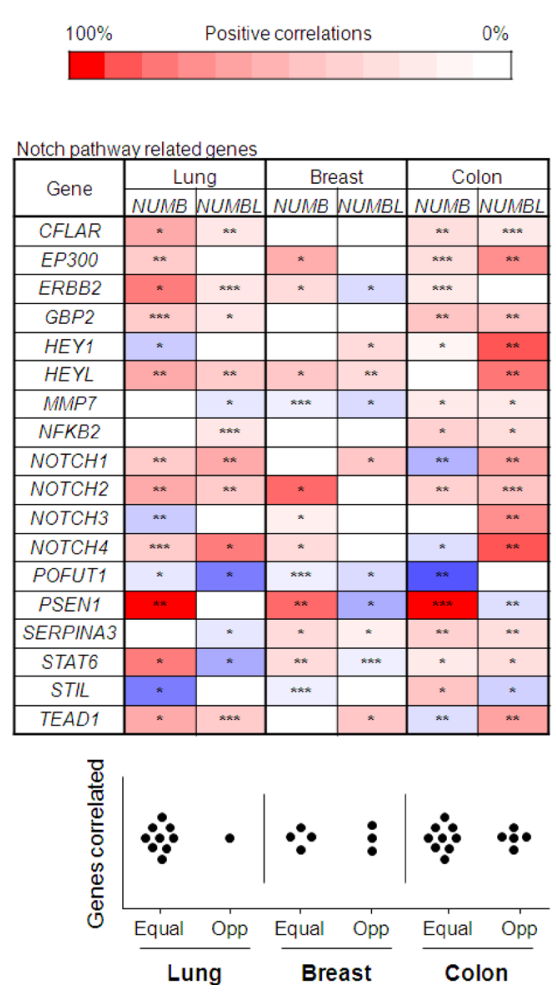
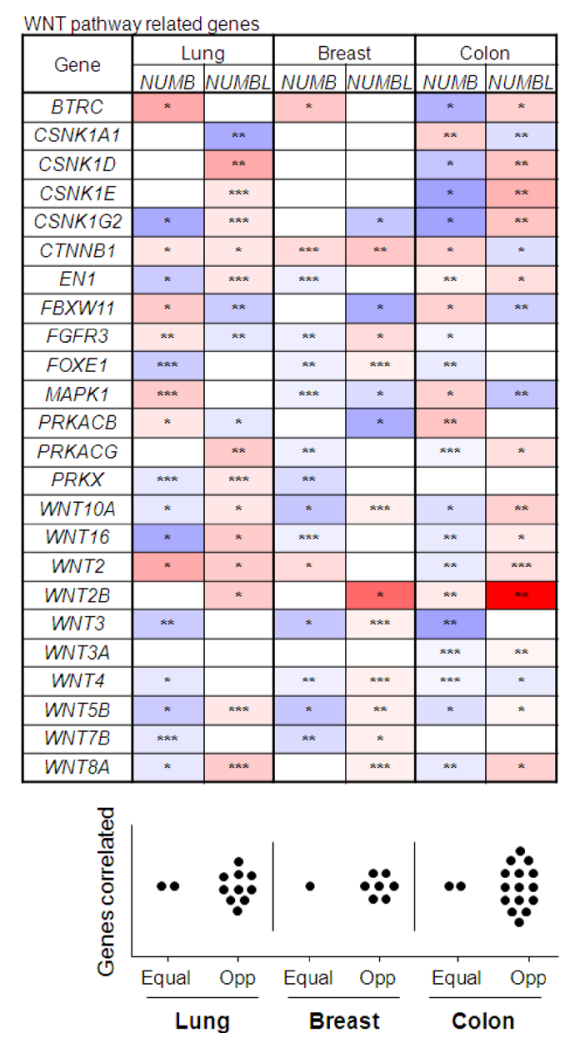
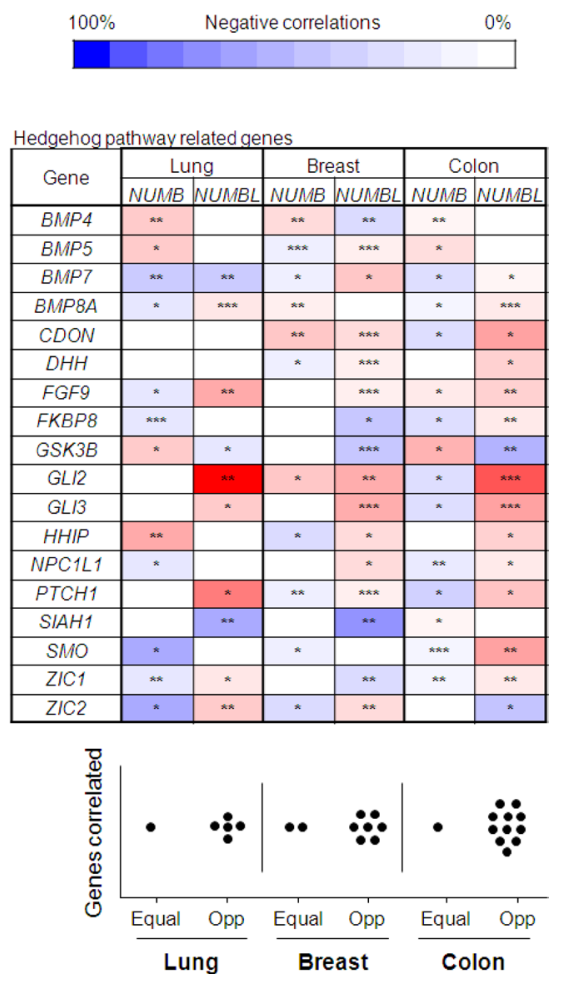

Figure 5: Upper figure: Notch, Hedgehog and WNT pathway genes with an opposite correlation for $N U M B$ and NUMBL. Statistical differences, obtained from gene correlations, are the followings: ${ }^{*}=p<0.05 ;{ }^{* *}=p<0.01 ;{ }^{* * *}=p<0.001$. Bottom figure: Graph representative of the genes equally regulated vs. opposite regulated for each pathway in breast, lung and colon tumors. 
have different effects on the signaling pathways (Figure 8). Since the phenotypic effect of $N U M B$ and $N U M B L$ downregulation is similar, inducing CSC-like properties, this finding suggest a hierarchy of Stem Cell pathways in which Notch pathway activation predominates over the other affected pathways, inducing an increase in stemness due to the lower levels of NUMB or NUMBL.

A possible explanation for the different effects on different pathways, correlating genes to either an oncogene or a tumor suppressor, may be plausible if we consider recent results correlating the alternative splicing of $N U M B$ in tumors. p72/p71 NUMB isoforms have been shown to be induced by RAS-ERK signaling in breast and lung cancer [49]. An analysis of all NUMB and NUMBL protein isoforms has shown that only NUMB p72/p66 exhibits the entire sequence of the PTB domain, with the absence of an 11-amino-acid sequence starting after Ala67 in the other two isoforms, $\mathrm{p} 71 / \mathrm{p} 65$. In addition, only p72/p71 exhibits the 48-amino-acid sequence that corresponds to exon 9, which has been linked to an increase in tumorigenic properties [49]. NUMBL also lacks this region, although protein homology between NUMB and NUMBL in this region is low, sharing less than $30 \%$ of the sequence between Ser373 and Leu574 from NUMBL. Thus, it is plausible that the NUMB and NUMBL isoforms are essential in terms of their different regulatory effects on the properties of cancer.

Expression data from public datasets, although a powerful tool to obtain useful information, may not detect
A
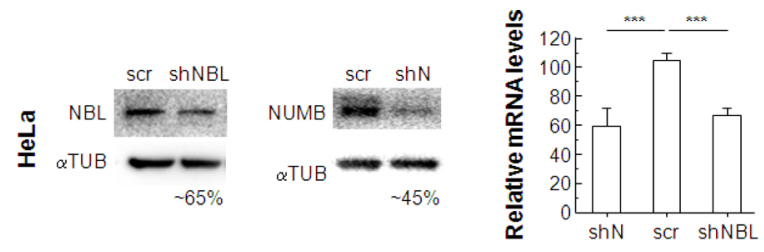

B
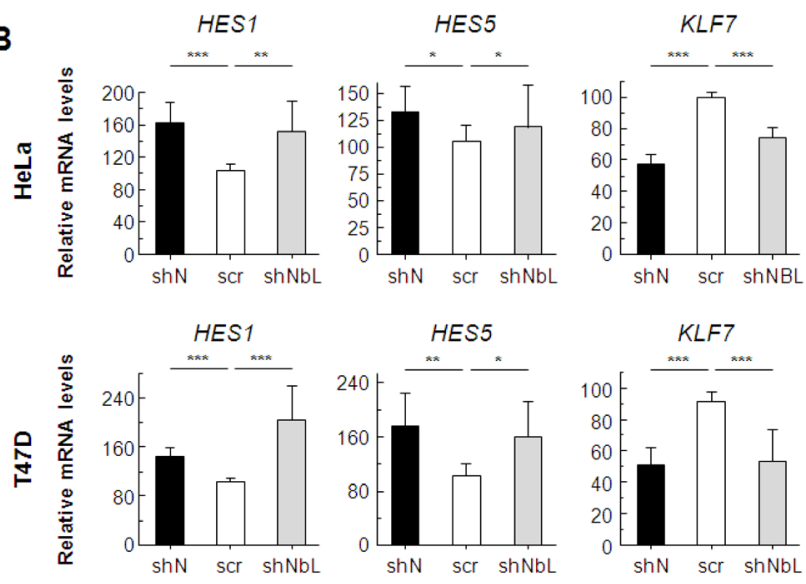

D
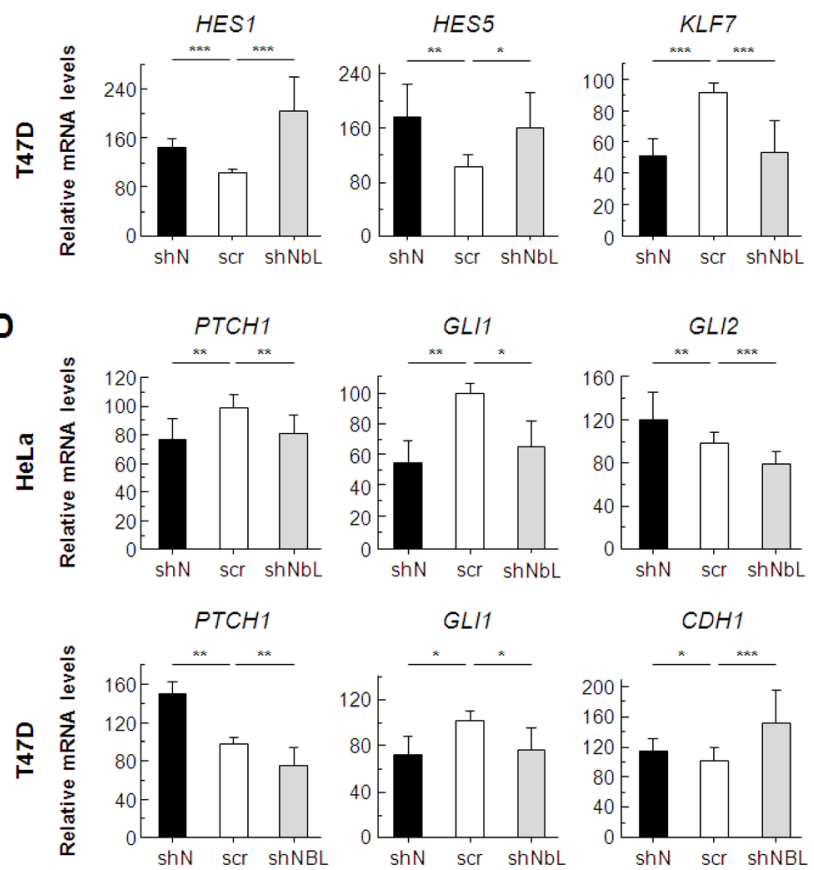

C
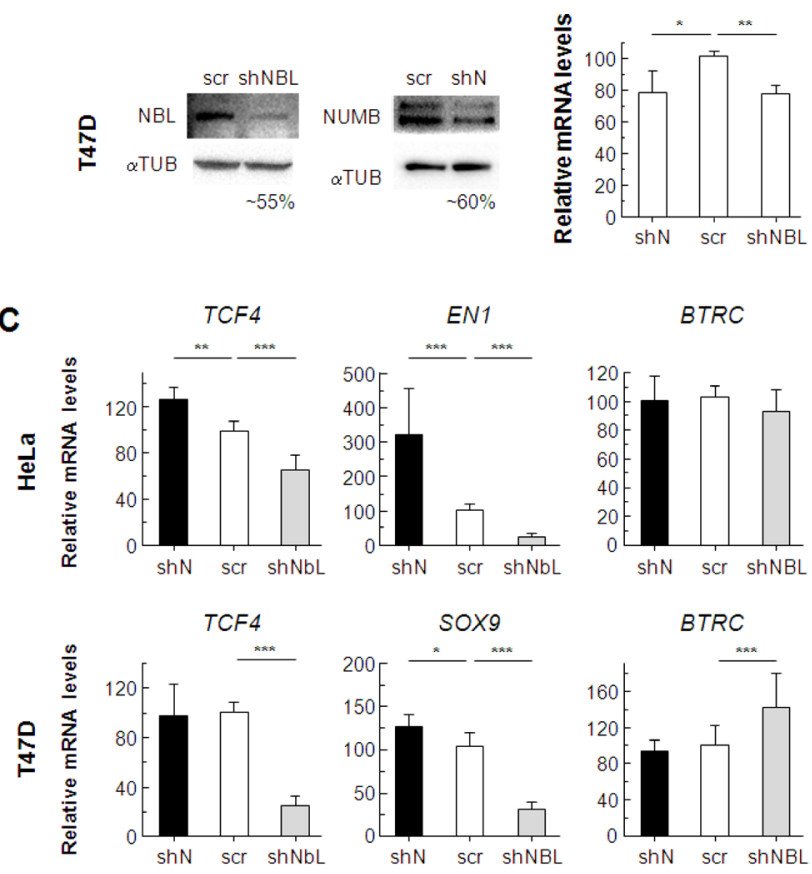

E
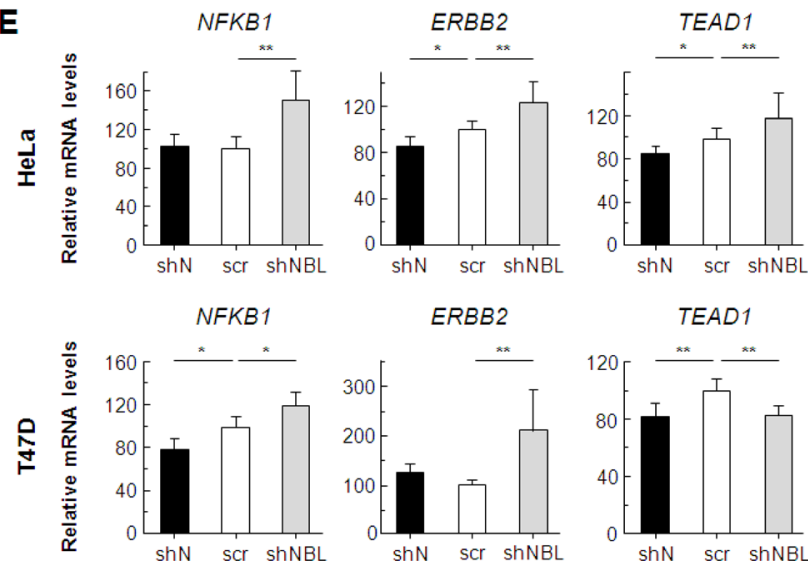

Figure 6: (A) Transfection of HeLa or T47D cells with $N U M B$ or $N U M B L$-shRNA plasmids induces a decrease in protein and mRNA expression, as detected by WB and qPCR. (B) $N U M B$ and $N U M B L$ downregulation modifies mRNA levels of Notch pathway-related genes. (C) $N U M B$ and $N U M B L$ downregulation modify the expression of genes in the WNT pathway in an opposite manner. (D) Changes in Hedgehog related genes due to $N U M B$ or $N U M B L$ downregulation. (E) $N U M B$ or $N U M B L$ downregulation results in opposite changes in gene expression of the NFKB, ERBB/HER and Hippo pathways. All experiments were repeated a minimum of three independent times. The Student's $T$ test was used to determine significant differences $\left({ }^{*}=p<0.05 ;{ }^{* *}=p<0.01 ;{ }^{* * *}=p<0.001\right)$. 
alternative splicing. Thus, although both NUMB and NUMBL are considered tumor suppressors, an increment in the expression of at least $N U M B$ cannot be assumed to lead to an increase in a tumor suppressor gene due to the observed alternative splicing. In accordance with this hypothesis, $N U M B$ levels are higher in astrocytomas and cervical squamous carcinoma cells, and the positive effect of NUMBL over SHH signaling can also promote tumor progression [23, 33, 34, 49]. Therefore, explorations of the effects of NUMB and NUMBL should not only consider the expression of both genes individually but also the relative levels of their different isoforms.

Alternatively, these differences might be derived from differences in the affinity of NUMB/NUMBL for its targets. In agreement with the previous hypothesis, NICD1 and NICD2, the processed intracellular domains of Notch1 and Notch2, showed similar effects over HES1 induction, while transcription of sequences upon the
A
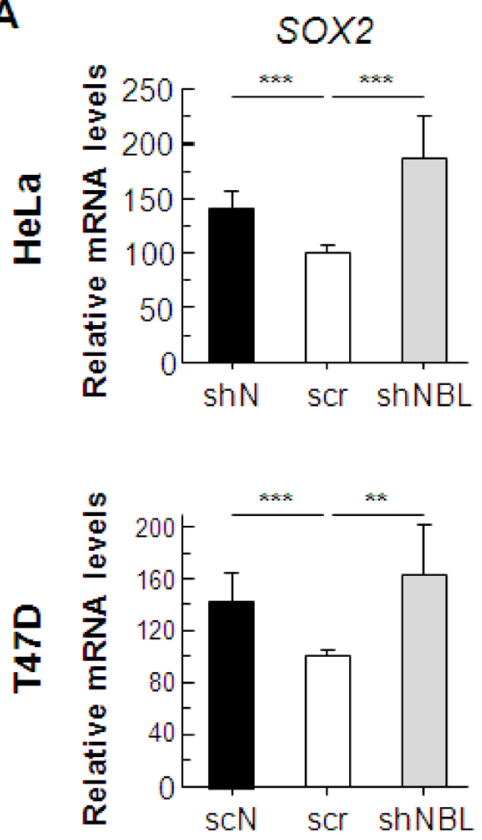

B
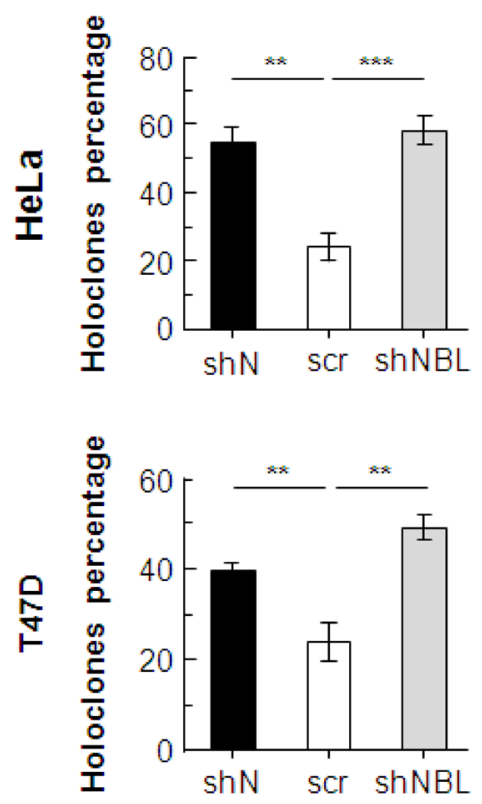

BMI1
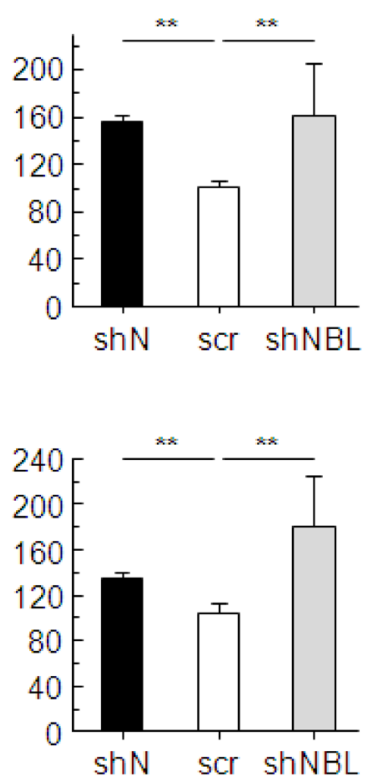

C
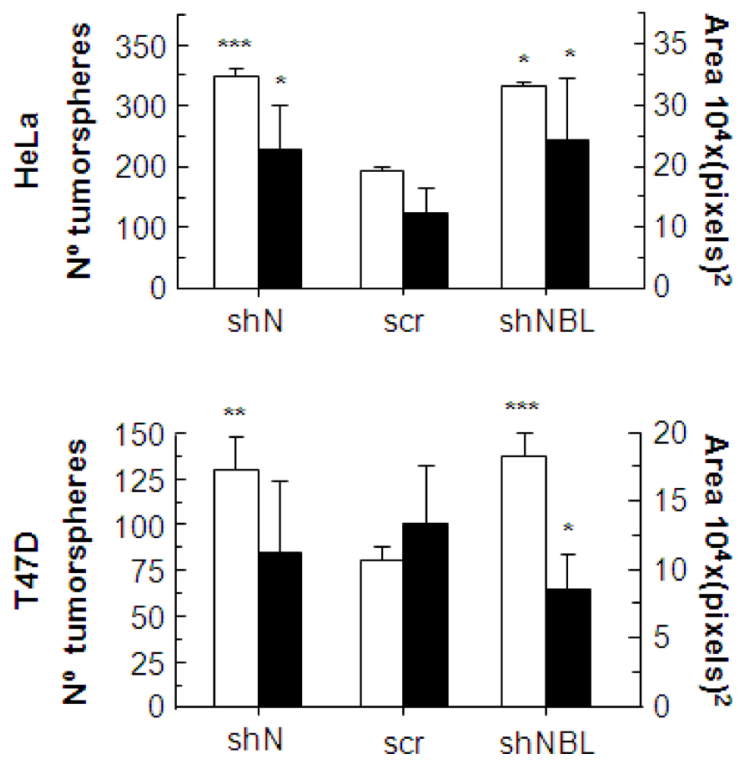

Figure 7: (A) Changes in the expression of genes related to stem cell properties due to $N U M B$ or $N U M B L$ downregulation. (B) Holoclone percentages are increased as a consequence of $N U M B$ or $N U M B L$ downregulation. (C) The number and area of tumorspheres are modified due to $N U M B$ or $N U M B L$ downregulation. All experiments were repeated a minimum of three independent times. The Student's $T$ test was used to determine significant differences $\left({ }^{*}=p<0.05 ;{ }^{* *}=p<0.01 ;{ }^{* * *}=p<0.001\right)$. 
four tandem CSL binding sites, typical of the promoters of other genes, were different [72]. In addition, the four NICD domains have been shown to be targets for different post-translational modifications, from ubiquitination to acetylation. The latter is particularly important because NICD1 acetylation has been shown to provide protection against degradation [34, 73]. Therefore, it is possible that NUMB and NUMBL preferentially target a different NICD member (not acetylated), explaining the observed differences in gene expression. In addition, Notch receptors have been shown to be differentially affected by Numb in mice [74]. Considering the presence of four different NUMB isoforms, NUMBL, the four Notch receptors and Notch's multiple post-translational modifications, a very complex scenario can be envisioned that still requires a great deal of research to disentangle.

In summary, these results indicated that NUMB and NUMBL could differentially modulate different signaling pathways in a very different fashion, indicating that, despite the previously observed exclusivity, the full redundancy does not exist between these two genes. Only in the Notch pathway, equal regulation is maintained. However, the similar phenotypic effect is observed under the downregulation of each protein independently, suggesting that Notch pathway regulation may predominate hierarchically in the regulation of the CSC phenotype.

\section{MATERIALS AND METHODS}

\section{Bioinformatics analysis}

To determine the correlation between $N U M B$ and $N U M B L$ genes in acute amyloid leukemia (AML), acute lymphoblastic leukemia (ALL), chronic lymphocytic leukemia (CLL), ovarian, colon/rectal, lung, renal, breast, neuroblastoma and esophagus tumors, a total of 95 different databases and 19 normal datasets were analyzed using R2 software (Genomics Analysis and Visualization Platform, http://r2.amc.nl). All datasets are freely available at the R2 webpage. To perform these correlations, the 209073 _s_at probe was used for $N U M B$ and the 242195 x at for $N U M B L$. In addition, for $N U M B$ vs. $N U M B L$ heat maps, we used TCGA datasets for Colon Adenocarcinoma and Kidney Renal Clear Cell Carcinomas, Bild dataset for tumor lung (GSE3141), and the EXPO dataset for breast and endometrium tumors (GSE2109).

To analyze the methylation state of $N U M B$ and $N U M B L$ in human samples, we used the TCGA Wanderer resource (http://maplab.imppc.org/wanderer/) [42], using the datasets for Breast Invasive Carcinoma, Colon Adenocarcinoma, Rectum Adenocarcinoma, Lung Adenocarcinoma, Lung Squamous Cell Carcinoma and Cervical squamous cell carcinoma and endocervical adenocarcinoma. Only CG probes with beta value higher than 0.1 were considered for the analysis.

To identify genes correlated with $N U M B$ and $N U M B L$, a total of 28 databases of different tumors (breast, lung, colon and cervix; Supplementary Table 1) were analyzed using R2 software. All datasets are freely available at the R2 webpage. We looked for correlations using probles 209073_s_at (NUMB) or 242195_x_at $(N U M B L)$ for all Affymetrix datasets. For the Budinska dataset, we used the ADXCRIH.2944.C1_at probe for $N U M B$ and the ADXCRAG_AF015041_at probe for $N U M B L$, while for the TCGA datasets, we used the NUMB_ 8650 probe for NUMB and the NUMBL_9253 probe for $N U M B L$. In all cases, we established a $p$-value lower than 0.05 to identify significant differences.

From the list of correlated genes, we separated genes that were positively correlated to NUMB or NUMBL from genes that were negatively correlated to NUMB or NUMBL, generating two gene lists for each database and gene. Next, we searched for genes that were

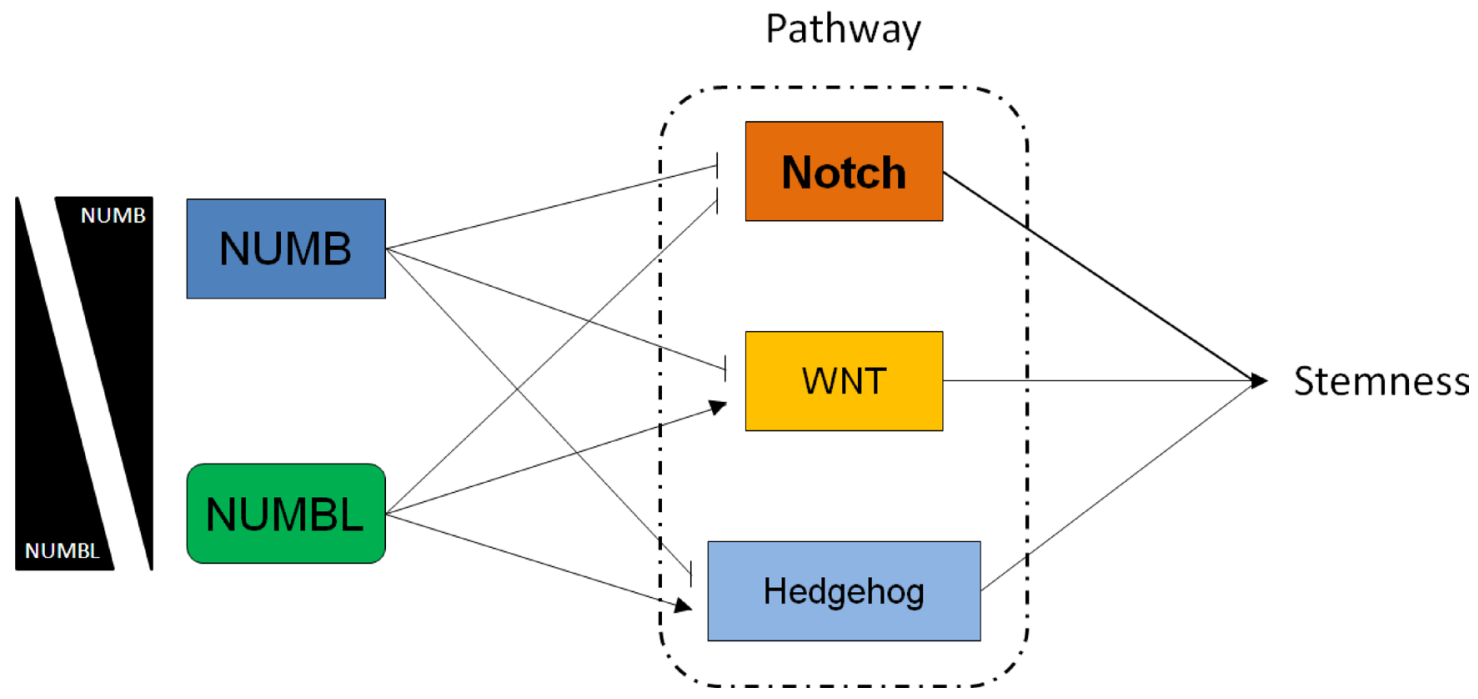

Figure 8: NUMB and NUMBL affect Notch, WNT and Hedgehog pathways differentially. 
highly represented among the different datasets. Thus, we established a cutoff for each gene of appearing at least in two different databases in cervical cancer, three different databases in lung cancer, four different databases in breast cancer and six different databases in colon cancer. We thereby generated four groups of genes that were commonly negatively or positively correlated to NUMB or NUMBL. To generate a Venn diagram to identify common genes that were correlated to NUMB or NUMBL for all tumors, we used the tool Venny [75].

To identify the pathways or Gene Ontology (GO) terms linked to genes that were positively or negatively correlated to NUMB or NUMBL, we used enrichment analysis from the Gene Ontology consortium webpage (http://geneontology.org/page/go-enrichment-analysis). In addition, we used Reactome (https://reactome.org/) to find altered pathways correlated to NUMB or NUMBL. In both cases, we only take into account genes with a $p$-value lower than 0.05 . For Reactome pathways, in addition, we only considered pathways with a FDR lower than 0.05 .

To detect correlations between $N U M B L$ and genes related to the Notch, WNT or Hedgehog pathways, we used the same previously considered 28 databases of different tumors (breast, lung and colon; Supplementary Table 1), fixing a $p$-value lower than 0.05 to identify statistically significant correlations.

\section{Cell lines and cellular assays}

T47D and HeLa cells were obtained from the European Collection of Authenticated Cell Cultures (ECACC) commercial repository at the beginning of this study. No further authentication was performed for these cell lines. Cells were maintained in DMEM (Sigma) supplemented with 10\% FBS (Life Technologies), penicillin, streptomycin, and fungizone. To downregulate $N U M B$ and $N U M B L$ expression, we used shRNA plasmids from Origene (TR311064, TR311063, Rockville, MD) as previously described $[17,48]$. All transfected cells were selected with $1 \mu \mathrm{g} \mathrm{mL}-1$ of puromycin.

\section{Protein isolation and western blot analysis}

Protein extracts for Western blot analysis were obtained as described previously [17]. For Western blot detection, we used NUMB (ab4147, Abcam, $1 \mu \mathrm{g} / \mathrm{mL}$ ) and NUMBL (ab37500, Abcam, $1 \mu \mathrm{g} / \mathrm{mL}$ ) antibodies. $\alpha$-Tubulin (T9026, Sigma) was used as a control. Horseradish peroxidase-labeled rabbit anti-mouse (ab97046, Abcam, diluted 1:5,000) and goat anti-rabbit (ab97051, Abcam, diluted 1:5,000) secondary antibodies were used.

\section{Analysis of gene transcription}

Total RNA was purified as described previously [17]. To detect changes in gene expression, we used the following probes, all from Life Technologies: NUMB (Hs01105433 m1), NUMBL (Hs00191080_m1), HES1 (Hs00172878_ m1), HES5 (Hs01387463_g1), KLF7 (Hs00748636_s1), NFKB1 (Hs00765730_m1), ERBB2 (Hs01001580_m1), TEAD1 (Hs00173359_m1), TCF4 (Hs00162613_m1), EN1 (Hs00154977_m1), BTRC (Hs00182707_m1), SOX9 (Hs01001343_g1), PTCH1 (Hs00181117_m1), GLI1 (Hs01110766_m1), GLI2 (Hs01119974_m1), CDH1 (Hs01023895_m1), SOX2 (Hs01053049_s1), BMI1 (Hs00995536_m1), NANOG (Hs0426036̄_g1) and GAPDH (Hs03929097_g1). Quantitative PCR was performed as described previously [17].

\section{ACKNOWLEDGMENTS AND FUNDING}

The authors thank Ana Rojas and Ildefonso Cases for the help with statistical analyses. This work was supported by grants from the Spanish Ministry of Economy and Competitivity, Plan Estatal de I+D+I 2013-2016, ISCIII (Fis: PI15/00045) and CIBER de Cáncer (CB16/12/00275), co-funded by FEDER from Regional Development European Funds (European Union), Consejería de Ciencia e Innovación (CTS-1848) and Consejería de Salud of the Junta de Andalucía (PI0096-2014). Especial thanks to the AECC Foundation for supporting this work. This work has also been made possible by the Fundación BBVA.

\section{CONFLICTS OF INTEREST}

The authors declare no conflicts of interest.

\section{REFERENCES}

1. Wang S, Younger-Shepherd S, Jan LY, Jan YN. Only a subset of the binary cell fate decisions mediated by Numb/ Notch signaling in Drosophila sensory organ lineage requires Suppressor of Hairless. Development. 1997; 124:4435-4446.

2. Nishimura T, Kaibuchi K. Numb Controls Integrin Endocytosis for Directional Cell Migration with aPKC and PAR-3. Developmental Cell. 2007; 13:15-28.

3. Santolini E, Puri C, Salcini AE, Gagliani MC, Pelicci PG, Tacchetti C, Di Fiore PP. Numb Is an Endocytic Protein. The Journal of Cell Biology. 2000; 151:1345-1352.

4. McGill MA, McGlade CJ. Mammalian Numb Proteins Promote Notch1 Receptor Ubiquitination and Degradation of the Notch1 Intracellular Domain. Journal of Biological Chemistry. 2003; 278:23196-23203.

5. Rasin MR, Gazula VR, Breunig JJ, Kwan KY, Johnson MB, Liu-Chen S, Li HS, Jan LY, Jan YN, Rakic P, Sestan N. Numb and Numbl are required for maintenance of cadherinbased adhesion and polarity of neural progenitors. Nat Neurosci. 2007; 10:819-827. 
6. Petersen PH, Zou K, Hwang JK, Jan YN, Zhong W. Progenitor cell maintenance requires numb and numblike during mouse neurogenesis. Nature. 2002; 419:929-934.

7. Petersen PH, Zou K, Krauss S, Zhong W. Continuing role for mouse Numb and Numbl in maintaining progenitor cells during cortical neurogenesis. Nat Neurosci. 2004; 7:803-811.

8. Uemura T, Shepherd S, Ackerman L, Jan LY, Jan YN. Numb, a gene required in determination of cell fate during sensory organ formation in Drosophila embryos. Cell. 1989; 58:349-360.

9. Zhong W, Jiang MM, Weinmaster G, Jan LY, Jan YN. Differential expression of mammalian Numb, Numblike and Notch1 suggests distinct roles during mouse cortical neurogenesis. Development. 1997; 124:1887-1897.

10. Zhong W, Feder JN, Jiang MM, Jan LY, Jan YN. Asymmetric Localization of a Mammalian Numb Homolog during Mouse Cortical Neurogenesis. Neuron. 1996; 17:43-53.

11. Wakamatsu Y, Maynard TM, Jones SU, Weston JA. NUMB Localizes in the Basal Cortex of Mitotic Avian Neuroepithelial Cells and Modulates Neuronal Differentiation by Binding to NOTCH-1. Neuron. 1999; 23:71-81.

12. Zilian O, Saner C, Hagedorn L, Lee HY, Säuberli E, Suter U, Sommer L, Aguet M. Multiple roles of mouse Numb in tuning developmental cell fates. Current Biology. 2001; 11:494-501.

13. Grisanti L, Corallini S, Fera S, Muciaccia B, Stefanini M, Witke W, Vicini E. Inactivation of Numb and Numblike in spermatogonial stem cells by cell-permeant Cre recombinase. Differentiation. 2009; 78:131-136.

14. Hirai M, Arita Y, McGlade CJ, Lee KF, Chen J, Evans SM. Adaptor proteins NUMB and NUMBL promote cell cycle withdrawal by targeting ERBB2 for degradation. The Journal of Clinical Investigation. 2017; 127:569-582.

15. Pece S, Confalonieri S, Romano PR, Di Fiore PP. NUMBing down cancer by more than just a NOTCH. Biochim Biophys Acta. 2011; 1815:26-43.

16. Amson R, Karp JE, Telerman A. Lessons from tumor reversion for cancer treatment. Current Opinion in Oncology. 2013; 25:59-65.

17. García-Heredia JM, Verdugo Sivianes EM, Lucena-Cacace A, Molina-Pinelo S, Carnero A. Numb-like (NumbL) downregulation increases tumorigenicity, cancer stem cell-like properties and resistance to chemotherapy. Oncotarget. 2016; 7:63611-63628. https://doi.org/10.18632/oncotarget.11553.

18. Colaluca IN, Tosoni D, Nuciforo P, Senic-Matuglia F, Galimberti V, Viale G, Pece S, Di Fiore PP. NUMB controls p53 tumour suppressor activity. Nature. 2008; 451:76-80.

19. Juven-Gershon T, Shifman O, Unger T, Elkeles A, Haupt Y, Oren M. The Mdm2 Oncoprotein Interacts with the Cell Fate Regulator Numb. Mol Cell Biol. 1998; 18:3974-3982.
20. Frise E, Knoblich JA, Younger-Shepherd S, Jan LY, Jan YN. The Drosophila Numb protein inhibits signaling of the Notch receptor during cell-cell interaction in sensory organ lineage. Proc Natl Acad Sci U S A. 1996; 93:11925-11932.

21. Gho M, Lecourtois M, Geraud G, Posakony JW, Schweisguth F. Subcellular localization of Suppressor of Hairless in Drosophila sense organ cells during Notch signalling. Development. 1996; 122:1673-1682.

22. Flores AN, McDermott N, Meunier A, Marignol L. NUMB inhibition of NOTCH signalling as a therapeutic target in prostate cancer. Nat Rev Urol. 2014; 11:499-507.

23. Hwang WL, Jiang JK, Yang SH, Huang TS, Lan HY, Teng HW, Yang CY, Tsai YP, Lin CH, Wang HW, Yang MH. MicroRNA-146a directs the symmetric division of Snaildominant colorectal cancer stem cells. Nat Cell Biol. 2014; 16:268-280.

24. Kang Y, Ding M, Tian G, Guo H, Wan Y, Yao Z, Li B, Lin D. Overexpression of Numb suppresses tumor cell growth and enhances sensitivity to cisplatin in epithelioid malignant pleural mesothelioma. Oncology Reports. 2013; 30:313-319.

25. Pece S, Serresi M, Santolini E, Capra M, Hulleman E, Galimberti V, Zurrida S, Maisonneuve P, Viale G, Di Fiore PP. Loss of negative regulation by Numb over Notch is relevant to human breast carcinogenesis. The Journal of Cell Biology. 2004; 167:215-221.

26. Westhoff B, Colaluca IN, D'Ario G, Donzelli M, Tosoni D, Volorio S, Pelosi G, Spaggiari L, Mazzarol G, Viale G, Pece S, Di Fiore PP. Alterations of the Notch pathway in lung cancer. Proc Natl Acad Sci U S A. 2009; 106:22293-22298.

27. Maiorano E, Favia G, Pece S, Resta L, Maisonneuve P, Di Fiore PP, Capodiferro S, Urbani U, Viale G. Prognostic Implications of Numb Immunoreactivity in Salivary Gland Carcinomas. International Journal of Immunopathology and Pharmacology. 2007; 20:779-789.

28. Marino S. Medulloblastoma: developmental mechanisms out of control. Trends in Molecular Medicine. 2005; 11:17-22.

29. Sima J, Zhang B, Yu Y, Sima X, Mao Y. Overexpression of Numb suppresses growth, migration, and invasion of human clear cell renal cell carcinoma cells. Tumor Biology. 2015; 36:2885-2892.

30. Yingjie L, Jian T, Changhai Y, Jingbo L. Numblike regulates proliferation, apoptosis, and invasion of lung cancer cell. Tumor Biology. 2013; 34:2773-2780.

31. Tao T, Cheng C, Ji Y, Xu G, Zhang J, Zhang L, Shen A. Numbl inhibits glioma cell migration and invasion by suppressing TRAF5-mediated NF- $\kappa$ B activation. Molecular Biology of the Cell. 2012; 23:2635-2644.

32. Liu L, Lanner F, Lendahl U, Das D. Numblike and Numb differentially affect p53 and Sonic Hedgehog signaling. Biochemical and Biophysical Research Communications. 2011; 413:426-431. 
33. Chen H, Chen X, Ye F, Lu W, Xie X. Symmetric Division and Expression of Its Regulatory Gene Numb in Human Cervical Squamous Carcinoma Cells. Pathobiology. 2009; 76:149-154.

34. Yan B, Omar FM, Das K, Ng WH, Lim C, Shiuan K, Yap CT, Salto-Tellez M. Characterization of Numb expression in astrocytomas. Neuropathology. 2008; 28:479-484.

35. Rajendran D, Zhang Y, Berry DM, McGlade CJ. Regulation of Numb isoform expression by activated ERK signaling. Oncogene. 2016; 35:5202-5213.

36. Spana EP, Doe CQ. Numb Antagonizes Notch Signaling to Specify Sibling Neuron Cell Fates. Neuron. 1996; 17:21-26.

37. Yang J, Bücker S, Jungblut B, Böttger T, Cinnamon Y, Tchorz J, Müller M, Bettler B, Harvey R, Sun QY, Schneider A, Braun T. Inhibition of Notch2 by Numb/ Numblike controls myocardial compaction in the heart. Cardiovascular Research. 2012; 96:276-285.

38. Gu Y, Masiero M, Banham AH. Notch signaling: its roles and therapeutic potential in hematological malignancies. Oncotarget. 2016; 7:29804-29823. https://doi.org/10.18632/ oncotarget. 7772 .

39. Hassan WA, Yoshida R, Kudoh S, Motooka Y, Ito T. Evaluation of role of Notch3 signaling pathway in human lung cancer cells. Journal of cancer research and clinical oncology. 2016; 142:981-993.

40. Giachino C, Boulay JL, Ivanek R, Alvarado A, Tostado C, Lugert S, Tchorz J, Coban M, Mariani L, Bettler B, Lathia J, Frank S, Pfister S, et al. A Tumor Suppressor Function for Notch Signaling in Forebrain Tumor Subtypes. Cancer cell. 2015; 28:730-742.

41. Bonyadi Rad E, Hammerlindl H, Wels C, Popper U, Ravindran Menon D, Breiteneder H, Kitzwoegerer M, Hafner C, Herlyn M, Bergler H, Schaider H. Notch4 signaling induces a mesenchymal-epithelial-like transition in melanoma cells to suppress malignant behaviors. Cancer research. 2016; 76:1690-1697.

42. Díez-Villanueva A, Mallona I, Peinado MA. Wanderer, an interactive viewer to explore DNA methylation and gene expression data in human cancer. Epigenetics \& Chromatin. $2015 ; 8: 22$.

43. Ming L. The MCM Complex: Its Role in DNA Replication and Implications for Cancer Therapy. Current Cancer Drug Targets. 2005; 5:365-380.

44. Kyriazis GA, Wei Z, Vandermey M, Jo DG, Xin O, Mattson MP, Chan SL. Numb Endocytic Adapter Proteins Regulate the Transport and Processing of the Amyloid Precursor Protein in an Isoform-dependent Manner: Implications for Alzheimer disease pathogenesis. The Journal of Biological Chemistry. 2008; 283:25492-25502.

45. Shao X, Liu Y, Yu Q, Ding Z, Qian W, Zhang L, Zhang J, Jiang N, Gui L, Xu Z, Hong Y, Ma Y, Wei Y, et al. Numb regulates vesicular docking for homotypic fusion of early endosomes via membrane recruitment of Mon1b. Cell Res. 2016; 26:593-612.

46. Le Roux I, Konge J, Le Cam L, Flamant P, Tajbakhsh S. Numb is required to prevent $\mathrm{p} 53$-dependent senescence following skeletal muscle injury. Nature Communications. 2015; 6:8528.

47. Zhou L, Ma Q, Shi H, Huo K. NUMBL interacts with TRAF6 and promotes the degradation of TRAF6. Biochemical and Biophysical Research Communications. 2010; 392:409-414.

48. García-Heredia JM, Lucena-Cacace A, Verdugo-Sivianes EM, Perez M, Carnero A. The cargo protein MAP17 (PDZK1IP1) regulates the cancer stem cell pool activating the Notch pathway by abducting NUMB. Clinical Cancer Research. 2017; 23:3871-3883.

49. Hoesel B, Schmid JA. The complexity of NF-kB signaling in inflammation and cancer. Molecular Cancer. 2013; 12:86-86.

50. Arteaga CL. ERBB receptors in cancer: signaling from the inside. Breast Cancer Research. 2011; 13:304.

51. Santucci M, Vignudelli T, Ferrari S, Mor M, Scalvini L, Bolognesi ML, Uliassi E, Costi MP. The Hippo Pathway and YAP/TAZ-TEAD Protein-Protein Interaction as Targets for Regenerative Medicine and Cancer Treatment. Journal of Medicinal Chemistry. 2015; 58:4857-4873.

52. Chen L, Daley GQ. Molecular basis of pluripotency. Human Molecular Genetics. 2008; 17:R23-R27.

53. Moon JH, Heo JS, Kim JS, Jun EK, Lee JH, Kim A, Kim J, Whang KY, Kang YK, Yeo S, Lim HJ, Han DW, Kim DW, et al. Reprogramming fibroblasts into induced pluripotent stem cells with Bmi1. Cell Res. 2011; 21:1305-1315.

54. Jo A, Denduluri S, Zhang B, Wang Z, Yin L, Yan Z, Kang R, Shi LL, Mok J, Lee MJ, Haydon RC. The versatile functions of Sox9 in development, stem cells, and human diseases. Genes \& Diseases. 2014; 1:149-161.

55. Barrandon Y, Green H. Three clonal types of keratinocyte with different capacities for multiplication. Proc Natl Acad Sci U S A. 1987; 84:2302-2306.

56. Beaver CM, Ahmed A, Masters JR. Clonogenicity: Holoclones and Meroclones Contain Stem Cells. PLoS One. 2014; 9:e89834.

57. Gulino A, Di Marcotullio L, Screpanti I. The multiple functions of Numb. Experimental Cell Research. 2010; 316:900-906.

58. Hansson EM, Lendahl U, Chapman G. Notch signaling in development and disease. Seminars in Cancer Biology. 2004; 14:320-328.

59. Watt FM, Estrach S, Ambler CA. Epidermal Notch signalling: differentiation, cancer and adhesion. Current Opinion in Cell Biology. 2008; 20:171-179.

60. Xia X, Zhou B, Chen X, Shen H, Huang H, Jin Q, Zhang $\mathrm{S}$. Loss of Numbl protein in osteosarcoma is associated with metastasis and poor survival. International Journal of Clinical and Experimental Pathology. 2016; 9:2027-2032. 
61. Vaira V, Faversani A, Martin NM, Garlick DS, Ferrero S, Nosotti M, Kissil JL, Bosari S, Altieri DC. Regulation of Lung Cancer Metastasis by Klf4-Numb-like Signaling. Cancer research. 2013; 73:2695-2705.

62. Leong KG, Gao WQ. The Notch pathway in prostate development and cancer. Differentiation. 2008; 76:699-716.

63. Pierfelice TJ, Schreck KC, Eberhart CG, Gaiano N. Notch, Neural Stem Cells, and Brain Tumors. Cold Spring Harbor symposia on quantitative biology. 2008; 73:367-375.

64. Wang Z, Li Y, Banerjee S, Sarkar FH. Emerging Role of Notch in Stem Cells and Cancer. Cancer Letters. 2009; 279:8-12.

65. Zweidler-McKay PA. Notch signaling in pediatric malignancies. Current Oncology Reports. 2008; 10:459-468.

66. Hu YY, Zheng MH, Zhang R, Liang YM, Han H. Notch Signaling Pathway and Cancer Metastasis. In: Reichrath J, Reichrath S, eds. Notch Signaling in Embryology and Cancer. (New York, NY: Springer US), pp. 186-198.

67. Yumei L, Jia M, Xiujuan Q, Qiong W, Jun X, Lucio M, Fazlul HS, Zhiwei W. Regulation of EMT by Notch Signaling Pathway in Tumor Progression. Current Cancer Drug Targets. 2013; 13:957-962.

68. Mumm JS, Schroeter EH, Saxena MT, Griesemer A, Tian X, Pan DJ, Ray WJ, Kopan R. A Ligand-Induced Extracellular Cleavage Regulates gamma-Secretase-like Proteolytic Activation of Notch1. Molecular Cell. 2000; 5:197-206.

69. Bertrand FE, McCubrey JA, Angus CW, Nutter JM, Sigounas G. NOTCH and PTEN in prostate cancer. Advances in Biological Regulation. 2014; 56:51-65.
70. Mazumdar T, DeVecchio J, Agyeman A, Shi T, Houghton JA. The GLI genes as the molecular switch in disrupting Hedgehog signaling in colon cancer. Oncotarget. 2011; 2:638-645. https://doi.org/10.18632/oncotarget.310.

71. Schreck KC, Taylor P, Marchionni L, Gopalakrishnan V, Bar EE, Gaiano N, Eberhart CG. The Notch Target Hes1 Directly Modulates Gli1 Expression and Hedgehog Signaling: A Potential Mechanism of Therapeutic Resistance. Clinical cancer research. 2010; 16:6060-6070.

72. Schroeder MP, Gonzalez-Perez A, Lopez-Bigas N. Visualizing multidimensional cancer genomics data. Genome Med. 2013; 5:9.

73. Marcotullio LD, Ferretti E, Greco A, De Smaele E, Po A, Sico MA, Alimandi M, Giannini G, Maroder M, Screpanti I, Gulino A. Numb is a suppressor of Hedgehog signalling and targets Gli1 for Itch-dependent ubiquitination. Nat Cell Biol. 2006; 8:1415-1423.

74. Beres BJ, George R, Lougher EJ, Barton M, Verrelli BC, McGlade CJ, Rawls JA, Wilson-Rawls J. Numb regulates Notch1, but not Notch3, during myogenesis. Mechanisms of Development. 2011; 128:247-257.

75. Oliveros JC. Venny. An interactive tool for comparing lists with Venn's diagrams. http://bioinfogp.cnb.csic.es/tools/ venny/index.html (2007-2015). 